\title{
ON THE POLYNOMIAL VECTOR FIELDS ON $\mathbb{S}^{2}$
}

\author{
JAUME LLIBRE ${ }^{1}$ AND YULIN ZHAO ${ }^{2}$
}

\begin{abstract}
Let $\mathcal{X}$ be a polynomial vector field of degree $n$ on $M$. If $M=\mathbb{R}^{m}$, then the dynamics and the algebraic-geometric properties of the vector fields $\mathcal{X}$ 's have been studied intensively, mainly for the case $M=\mathbb{R}^{2}$, and specially when $n=2$. In this last case more than one thousand of papers have been published.

If $M=\mathbb{S}^{2}$ there are several papers dedicated to the study of the homogeneous polynomial vector field of degree $n$ on $\mathbb{S}^{2}$, mainly if $n=2$. But there are very few results on the non-homogeneous polynomial vector fields of degree $n$ on $\mathbb{S}^{2}$. In this paper we shall try to full a little this gap.
\end{abstract}

\section{Introduction AND STATEMENT OF the MAIN RESUlts}

Let $\mathbb{R}[x, y, z]$ be the ring of all polynomials in the variables $x, y$ and $z$ with real coefficients. The vector field

$$
\mathcal{X}=P(x, y, z) \frac{\partial}{\partial x}+Q(x, y, z) \frac{\partial}{\partial y}+R(x, y, z) \frac{\partial}{\partial z}
$$

is called a polynomial vector field of degree $n$ in $\mathbb{R}^{3}$ if $P, Q, R \in \mathbb{R}[x, y, z]$ and $n=\max \{\operatorname{deg} P, \operatorname{deg} Q, \operatorname{deg} R\}$. For simplicity sometimes we will write the vector field $\mathcal{X}$ simply as $\mathcal{X}=(P, Q, R)$.

The vector field (1) is a homogeneous polynomial vector field $\mathcal{X}$ of degree $n$ in $\mathbb{R}^{3}$ if $P, Q, R$ are homogeneous polynomials of degree $n$.

Let $f \in \mathbb{R}[x, y, z]$. The algebraic surface $f(x, y, z)=0$ is an invariant algebraic surface of $\mathcal{X}$ if there exists $K \in \mathbb{R}[x, y, z]$ such that

$$
\mathcal{X} f=P(x, y, z) \frac{\partial f}{\partial x}+Q(x, y, z) \frac{\partial f}{\partial y}+R(x, y, z) \frac{\partial f}{\partial z}=K(x, y, z) f(x, y, z) .
$$

As usual $\mathbb{S}^{2}$ denotes the 2-dimensional sphere $\left\{(x, y, z) \in \mathbb{R}^{3}: x^{2}+y^{2}+z^{2}=1\right\}$. A polynomial vector field $\mathcal{X}$ of degree $n$ on $\mathbb{S}^{2}$ is a polynomial vector field in $\mathbb{R}^{3}$ of degree $n$ such that restricted to $\mathbb{S}^{2}$ defines a vector field on $\mathbb{S}^{2}$, i.e. it must satisfy the equality

$$
x P(x, y, z)+y Q(x, y, z)+z R(x, y, z)=0
$$

for all $(x, y, z) \in \mathbb{S}^{2}$. In particular $\mathcal{X}$ is called a quadratic vector field on $\mathbb{S}^{2}$ if $n=2$.

There are other definitions of polynomial vector fields on the sphere $\mathbb{S}^{2}$. For instance, let $A, B$ and $C$ three polynomials of degree $n-1$, then by definition a polynomial vector field $\mathcal{X}$ on $\mathbb{S}^{2}$ of degree $n$ is $\mathcal{X}=(P, Q, R)=(x, y, z) \wedge(A, B, C)$. Of course this second definition implies our definition $x P+y Q+z R=0$, but not

2000 Mathematics Subject Classification. Primary 34C05, 34A34, 34C14.

Key words and phrases. Polynomial vector fields on the sphere, limit cycle, singular points, great circle. 
only in the points of $(x, y, z) \in \mathbb{S}^{2}$, also in all the points of $(x, y, z) \in \mathbb{R}^{3}$. We do not consider this definition in this paper.

The algebraic surface $f(x, y, z)=0$ defines an invariant algebraic curve $\{f=$ $0\} \cap \mathbb{S}^{2}$ of the polynomial vector field $\mathcal{X}$ on $\mathbb{S}^{2}$ if

(i) there exists $K \in \mathbb{R}[x, y, z]$ such that (2) holds, and

(ii) the intersection of the two surfaces $f(x, y, z)=0$ and $\mathbb{S}^{2}$ is transversal, i.e. for all points $(x, y, z) \in\{f=0\} \cap \mathbb{S}^{2}$, we have that $(x, y, z) \wedge$ $(\partial f / \partial x, \partial f / \partial y, \partial f / \partial z) \neq 0$, where wedge denotes the cross product vector of $\mathbb{R}^{3}$. In particular condition (ii) guarantees that the intersection of the two surfaces $\{f(x, y, z)=0\} \cap \mathbb{S}^{2}$ is a a curve, not a surface.

The algebraic curve $\{a x+b y+c z=0\} \cap \mathbb{S}^{2}$ is called a great circle on $\mathbb{S}^{2}$, where $a, b, c$ are real constants. Moreover a great circle is an invariant great circle of a polynomial vector field $\mathcal{X}$ of degree $n$ on $\mathbb{S}^{2}$ if it is an invariant algebraic surface of $\mathcal{X}$.

All the above definitions can be found in for instance in [1, 9, 10, 13].

The authors of the paper [9] studied homogeneous polynomial vector fields of degree 2 on $\mathbb{S}^{2}$. They determined the maximum number of invariant circles when such a vector field has finitely many invariant circles. The same authors found the upper bound for the number of invariant circles, invariant great circles of homogeneous polynomial vector fields of degree $n$ on $\mathbb{S}^{2}[10]$. It is proved in [11] that if a homogeneous polynomial vector field $\mathcal{X}$ of degree 2 on $\mathbb{S}^{2}$ has at least a non-hyperbolic singularity, then it has no limit cycle. They also give necessary and sufficient conditions for determining if a singularity of a homogeneous polynomial vector field $\mathcal{X}$ of degree 2 on $\mathbb{S}^{2}$ is a center, and characterize the global phase portrait of $\mathcal{X}$ modulo limit cycles.

Coleman [1] and Sharipov [13] studied homogeneous vector fields of degree $n$ in $\mathbb{R}^{3}$. They showed that any such vector fields induced naturally on $\mathbb{S}^{2}$ a tangent vector field. Camacho [2] proved some properties of the vector fields on $\mathbb{S}^{2}$ induced by homogeneous vector fields of degree 2 in $\mathbb{R}^{3}$, see also $[8,14,15]$.

We note that the above cited results are concerned with vector fields on $\mathbb{S}^{2}$ homogeneous, or which are induced by homogeneous polynomial vector fields of $\mathbb{R}^{3}$.

In what follows we shall use many basic definitions of the qualitative theory of differential equations as singular point, node, saddle, focus, center, antisaddles, period annulus, topological index or simply index of a singular point, contact point, periodic orbit, limit cycle, homoclinic loop, tranversal arc, topologically equivalent, ... All the precise definitions of these notions can be found, for instance, in [4]. A node is called proper if its two real eigenvalues are different.

Our main results are presented into the next two theorems. In the first we present results for polynomial vector fields on $\mathbb{S}^{2}$ of arbitrary degree, and in the second only for degree 2 .

Theorem 1. Let $\mathcal{X}$ be a polynomial vector field on $\mathbb{S}^{2}$ of degree $n$ having a finite number $s(\mathcal{X})$ of singular points in $\mathbb{S}^{2}$. Assume that $\mathcal{X}$ is given by (1).

(a) If one of the three polynomial systems $P=Q=x^{2}+y^{2}+z^{2}-1=0$, $Q=R=x^{2}+y^{2}+z^{2}-1=0$ or $R=P=x^{2}+y^{2}+z^{2}-1=0$, has finitely many solutions in $\mathbb{C} P^{3}$, then $s(\mathcal{X}) \leq 2 n^{2}$. 
(b) If $n \geq 2$, then $s(\mathcal{X}) \leq(2 n-2)(2 n-1)+1$; and if $n=1$, then $s(\mathcal{X}) \leq 2$.

(c) Assume that all the singular points of $\mathcal{X}$ has topological index 1 or -1 . If $n \geq 2$, then $s(\mathcal{X}) \leq(2 n-2)(2 n-1)$; and if $n=1$, then $s(\mathcal{X})=2$.

(d) Every great circle is either an invariant great circle of $\mathcal{X}$, or has at most $2 n$ contact points (including singular points) with the orbits of $\mathcal{X}$.

The singular points of the vector field $\mathcal{X}$ on $\mathbb{S}^{2}$ are included in the points solution of the polynomial system $P=Q=x^{2}+y^{2}+z^{2}-1=0$. By the multi-dimensional Bezout Theorem (see $[3,12]$ ), the number of solutions of this polynomial system in $\mathbb{C} P^{3}$ are either infinity, or less than $2 n^{2}$. This is the proof of statement (a) of Theorem 1 .

On the other hand it is important to note that there are polynomial vector fields $\mathcal{X}=(P, Q, R)$ on $\mathbb{S}^{2}$ for which the three polynomial systems of the statement (a) of Theorem 1 have infinitely many solutions in $\mathbb{C} P^{3}$, and consequently we cannot apply Bezout Theorem for bounding its number of singular points, but such polynomial vector fields can have finitely many singular points. An example of these kind of polynomial vector field on $\mathbb{S}^{2}$ is

$$
P=y z\left(1-3 x^{2}\right), \quad Q=x z\left(1-3 y^{2}\right), \quad R=x y\left(1-3 z^{2}\right) .
$$

Clearly every one of the three systems of statement (a) of Theorem 1 contains as solution one circle of the sphere, so Bezout Theorem does not provide any bound for the number of singular points. But the singular points of $\mathcal{X}$ on $\mathbb{S}^{2}$ are finite, namely $( \pm 1, \pm 1, \pm 1) / \sqrt{3},( \pm 1,0,0),(0, \pm 1,0)$ and $(0,0, \pm 1)$.

Theorem 2. Let $\mathcal{X}$ be a quadratic polynomial vector field on $\mathbb{S}^{2}$ having a finite number $s(\mathcal{X})$ of singular points of $\mathcal{X}$.

(a) Assume that all the singular points of $\mathcal{X}$ has topological index 1 or -1.

(a.1) The exact upper bound 6 for $s(\mathcal{X})$ is reached.

(a.2) $\mathcal{X}$ has at most 4 centers. This bound is reached for a convenient $\mathcal{X}$.

(a.3) $\mathcal{X}$ has at most 4 foci. This bound is reached for a convenient $\mathcal{X}$.

(a.4) $\mathcal{X}$ has at most 2 saddles. This bound is reached for a convenient $\mathcal{X}$.

(b) Assume that $\mathcal{X}$ has an invariant great circle, without loss of generality we can assume that it is $\mathbb{S}^{1}=\{z=0\} \cap \mathbb{S}^{2}$.

(b.1) The vector field $\mathcal{X}$ can be written as (1) with

$P(x, y, z)=a_{0}+a_{1} y-a_{0} x^{2}+a_{3} y^{2}+a_{4} z^{2}+a_{5} x y+a_{7} y z$,

$Q(x, y, z)=b_{0}-a_{1} x-\left(a_{5}+b_{0}\right) x^{2}-b_{0} y^{2}+b_{2} z^{2}-\left(a_{0}+a_{3}\right) x y-a_{7} x z$,

$R(x, y, z)=c_{0}-c_{0} x^{2}-c_{0} y^{2}-c_{0} z^{2}-\left(a_{0}+a_{4}\right) x z-\left(b_{0}+b_{2}\right) y z$.

(b.2) The great circle $\mathbb{S}^{1}$ is a periodic orbit of $\mathcal{X}$ if and only if

$$
\left(a_{0}+a_{3}\right)^{2}+a_{5}^{2}-a_{1}^{2}<0 .
$$

(b.3) The great circle $\mathbb{S}^{1}$ is a hyperbolic limit cycle if and only if (5) holds and

$$
\frac{a_{5}\left(a_{0}+a_{4}\right)+\left(a_{0}+a_{3}\right)\left(b_{0}+b_{2}\right)}{\sqrt{a_{1}^{2}-a_{5}^{2}-\left(a_{0}+a_{3}\right)^{2}}\left(\left|a_{1}\right|+\sqrt{a_{1}^{2}-a_{5}^{2}-\left(a_{0}+a_{3}\right)^{2}}\right)} \neq 0 .
$$

(b.4) If (5) holds and the right hand of (6) is equal to zero, and $\left(a_{0}+\right.$ $\left.a_{4}\right)^{2}+\left(b_{0}+b_{2}\right)^{2} \neq 0$, then the real parts of the two eigenvalues at every singular point of $\mathcal{X}$ is zero.

(c) A homoclinic loop of $\mathcal{X}$ does not contain any arc of a great circle. 
(d) There are non-homogeneous $\mathcal{X}$ having a great circle as a limit cycle.

(e) If $\mathcal{X}$ is homogeneous, then any great circle cannot be a periodic orbit of $\mathcal{X}$.

(f) There are $\mathcal{X}$ having the equator of $\mathbb{S}^{2}$ as a limit cycle and surrounding three singular point in one hemisphere and one singular point in the other.

(g) If $\mathcal{X}$ is homogeneous and has a hyperbolic saddle or a hyperbolic proper node, then there exists a great circle $\mathcal{C}$ such that either all orbits of $\mathcal{X}$ are transversal to $\mathcal{C}$, or $\mathcal{C}$ is an invariant great circle.

(h) There are $\mathcal{X}$ having two centers, and all the other orbits are periodic.

(i) There are $\mathcal{X}$ having two invariant great circles $\mathcal{C}_{1}$ and $\mathcal{C}_{2}$. Every one of the four connected component of $\mathbb{S}^{2} \backslash\left\{\mathcal{C}_{1} \cup \mathcal{C}_{2}\right\}$ is completely filled by a center and its period annulus.

The paper is structured as follows. In section 2 we introduce the notion of stereographic projection, that we shall use for proving many of our results. Theorems 1 and 2 will be proved in Sections 3 and 4, respectively.

We recall that Écalle [5] and Ilyashenko [7] proved independently that any analytic vector field on the sphere $\mathbb{S}^{2}$ has finitely many limit cycles. So in particular any polynomial vector field on $\mathbb{S}^{2}$ has a finite number of limit cycles.

\section{Stereographic Projection}

The stereographic projection has been used frequently in the study of polynomial vector fields on $\mathbb{S}^{2}$. Here we will follow [9] with small changes.

We identify $\mathbb{R}^{2}$ with the plane $a x+b y+c z=0$ where $a^{2}+b^{2}+c^{2}=1$. Suppose $c \neq 0$, then the points in $\mathbb{R}^{2}$ are denoted by $(u, v,-(a u+b v) / c)$. Let $\pi: \mathbb{R}^{2} \rightarrow$ $\mathbb{S}^{2} \backslash\{(a, b, c)\}$ be the diffeomorphism given by

$$
\begin{aligned}
\pi\left(u, v,-\frac{a u+b v}{c}\right) & =(x, y, z) \\
& =\left(\frac{a \lambda-2 c^{2}(a-u)}{\lambda}, \frac{b \lambda-2 c^{2}(b-v)}{\lambda}, \frac{c \lambda-2 c\left(c^{2}+a u+b v\right)}{\lambda}\right),
\end{aligned}
$$

where $\lambda=c^{2}\left(1+u^{2}+v^{2}\right)+(a u+b v)^{2}$. Therefore the stereographic projection $\pi^{-1}: \mathbb{S}^{2} \backslash\{(a, b, c)\} \rightarrow \mathbb{R}^{2}$ is defined by

$$
\begin{aligned}
\pi^{-1}(x, y, z) & =\left(u, v,-\frac{a u+b v}{c}\right) \\
& =\left(\frac{x-a(a x+b y+c z)}{1-(a x+b y+c z)}, \frac{y-b(a x+b y+c z)}{1-(a x+b y+c z)}, \frac{z-c(a x+b y+c z)}{1-(a x+b y+c z)}\right) .
\end{aligned}
$$

Through the stereographic projection $\pi^{-1}$ the polynomial system $\mathcal{X}$ on $\mathbb{S}^{2}$ becomes the differential system

$$
\begin{aligned}
& \dot{u}=\frac{1}{2 c^{2} \lambda^{n-1}}(\bar{P}+(u-a)(a \bar{P}+b \bar{Q}+c \bar{R})), \\
& \dot{v}=\frac{1}{2 c^{2} \lambda^{n-1}}(\bar{Q}+(v-b)(a \bar{P}+b \bar{Q}+c \bar{R})),
\end{aligned}
$$

on the plane $\mathbb{R}^{2}$, where $\bar{S}(u, v)=\lambda^{n} S(\pi(u, v,-(a u+b v) / c))$ and $S \in\{P, Q, R\}$. Let $t$ be the independent variable in the above differential system. Of course the 
dot denotes derivative with respect to $t$. Introducing the new independent variable $d s=d t /\left(2 c^{2} \lambda^{n-1}\right)$, we get the polynomial differential system

$$
\begin{aligned}
& \dot{u}=\bar{P}+(u-a)(a \bar{P}+b \bar{Q}+c \bar{R}), \\
& \dot{v}=\bar{Q}+(v-b)(a \bar{P}+b \bar{Q}+c \bar{R})) .
\end{aligned}
$$

Now the dot denotes derivative with respect to $s$. The planar vector field (8) will be called the stereographic projection of vector field $\mathcal{X}=(P, Q, R)$ at the point $(a, b, c) \in \mathbb{S}^{2}$.

In general we consider the stereographic projections at the points $(a, b, c)=$ $(0,0,1)$ and $(a, b, c)=(0,0,-1)$, and we denote their corresponding stereographic projections by $\pi_{1}$ and $\pi_{-1}$. These two projections form an atlas for the sphere $\mathbb{S}^{2}$.

Let

$$
\begin{aligned}
& P(x, y, z)=\sum_{i+j+k \leq n} p_{i j k} x^{i} y^{j} z^{k}, \\
& Q(x, y, z)=\sum_{i+j+k \leq n} q_{i j k} x^{i} y^{j} z^{k}, \\
& R(x, y, z)=\sum_{i+j+k \leq n}^{i j n} r_{i j k} x^{i} y^{j} z^{k} .
\end{aligned}
$$

If $(a, b, c)=(0,0,1)$, then system (8) becomes

$$
\begin{aligned}
& \dot{u}=\bar{P}(u, v)+u \bar{R}(u, v)=\mathcal{P}(u, v), \\
& \dot{v}=\bar{Q}(u, v)+v \bar{R}(u, v)=\mathcal{Q}(u, v),
\end{aligned}
$$

where

$$
\begin{aligned}
& \bar{P}(u, v)=\sum_{i+j+k \leq n} p_{i j k}(2 u)^{i}(2 v)^{j}\left(u^{2}+v^{2}-1\right)^{k}\left(u^{2}+v^{2}+1\right)^{n-i-j-k}, \\
& \bar{Q}(u, v)=\sum_{i+j+k \leq n}(2 u)^{i}(2 v)^{j}\left(u^{2}+v^{2}-1\right)^{k}\left(u^{2}+v^{2}+1\right)^{n-i-j-k}, \\
& \bar{R}(u, v)=\sum_{i+j+k \leq n}^{i+j+k} r_{i j k}(2 u)^{i}(2 v)^{j}\left(u^{2}+v^{2}-1\right)^{k}\left(u^{2}+v^{2}+1\right)^{n-i-j-k} .
\end{aligned}
$$

If $(a, b, c)=(0,0,-1)$, then system (8) writes

$$
\begin{aligned}
& \dot{u}=\widetilde{P}(u, v)-u \widetilde{R}(u, v), \\
& \dot{v}=\widetilde{Q}(u, v)-v \widetilde{R}(u, v),
\end{aligned}
$$

with

$$
\begin{aligned}
& \widetilde{P}(u, v)=\sum_{i+j+k \leq n} p_{i j k}(2 u)^{i}(2 v)^{j}\left(1-u^{2}-v^{2}\right)^{k}\left(u^{2}+v^{2}+1\right)^{n-i-j-k}, \\
& \widetilde{Q}(u, v)=\sum_{i+j+k \leq n} q_{i j k}(2 u)^{i}(2 v)^{j}\left(1-u^{2}-v^{2}\right)^{k}\left(u^{2}+v^{2}+1\right)^{n-i-j-k}, \\
& \widetilde{R}(u, v)=\sum_{i+j+k \leq n} r_{i j k}(2 u)^{i}(2 v)^{j}\left(1-u^{2}-v^{2}\right)^{k}\left(u^{2}+v^{2}+1\right)^{n-i-j-k} .
\end{aligned}
$$

In what follows we are going to study the properties of the polynomial vector fields $\mathcal{X}$ of degree $n$ on $\mathbb{S}^{2}$ by using the stereographic projections (10) and (12). 


\section{Proof of Theorem 1}

We shall use the next result.

Lemma 3. Let $\mathcal{X}$ be the polynomial vector field of degree $n$ on $\mathbb{S}^{2}$ given (1). Then the polynomial differential system (10) (resp. (12)) has degree at most $2 n$. Moreover it has degree at most $2 n-1$ if $(0,0,1)$ (resp. $(0,0,-1))$ is a singular point of $\mathcal{X}$.

Proof. We do the proof for system (10). Similar arguments prove the lemma for system (12).

We get from $(11)$ that $\operatorname{deg} \bar{P}(u, v) \leq 2 n, \operatorname{deg} \bar{Q}(u, v) \leq 2 n$. It follows from $(3)$ that

$$
2 u \bar{P}(u, v)+2 v \bar{Q}(u, v)+\left(u^{2}+v^{2}-1\right) \bar{R}(u, v)=0,
$$

which implies $\bar{R}(u, v)$ is a polynomial of degree at most $2 n-1$. Hence system (10) is a polynomial system of degree at most $2 n$.

It follows from (11) that the homogeneous parts of $\bar{P}(u, v)$ and $\bar{Q}(u, v)$ of degree $2 n$ are

$$
\left(\sum_{k=0}^{n} p_{00 k}\right)\left(u^{2}+v^{2}\right)^{n}, \quad\left(\sum_{k=0}^{n} q_{00 k}\right)\left(u^{2}+v^{2}\right)^{n}
$$

respectively.

By the Poincaré-Hopf Theorem every vector field on $\mathbb{S}^{2}$ has singular points, see for instance [4]. Suppose that $(0,0,1)$ is a singular point of $\mathcal{X}$. Then we have

$$
\sum_{k=0}^{n} p_{00 k}=0, \quad \sum_{k=0}^{n} q_{00 k}=0 .
$$

From (15) and (16) we get that $\bar{P}(u, v)$ and $\bar{Q}(u, v)$ are polynomials of degree at most $2 n-1$. Equation (14) implies that $\bar{R}(u, v)$ is a polynomial of degree at most $2 n-2$. Therefore system (10) has degree at most $2 n-1$ if $(0,0,1)$ is a singular point of $\mathcal{X}$ on $\mathbb{S}^{2}$.

Now we shall prove Theorem 1. We only need to prove statements (b), (c) and (d).

Proof of statement (b) of Theorem 1. Let $p$ be a singular point of $\mathcal{X}$. Doing a convenient rotation of $S O(3)$ we can assume that $p=(0,0,1)$. Let $(u, v)$ be a singular point of system (10). Then $\bar{P}(u, v)=-u \bar{R}(u, v), \bar{Q}(u, v)=-v \bar{R}(u, v)$. This together with equation (14) shows that $\bar{R}(u, v)=0$. Therefore the coordinates $(u, v)$ of singular points are determined by the equations $\bar{P}(u, v)=$ $\bar{Q}(u, v)=\bar{R}(u, v)=0$. Since we have shown in the proof of Lemma 3 that $\operatorname{deg} \bar{P}(u, v) \leq 2 n-1, \operatorname{deg} \bar{Q}(u, v) \leq 2 n-1, \operatorname{deg} \bar{R}(u, v) \leq 2 n-2$, by Bezout Theorem (see, for example, [6]) system (10) has at most $(2 n-2)(2 n-1)$ isolated singular points. Noting that $(0,0,1)$ is also a singular point, the polynomial vector field $\mathcal{X}$ of degree $n$ on $\mathbb{S}^{2}$ has at most $(2 n-2)(2 n-1)+1$ isolated singular points.

If $n=1$ and $(0,0,1)$ is a singular point of $\mathcal{X}$, then $R(x, y, z) \equiv 0$. By direct computation we known that $\mathcal{X}$ has at most 2 singular points if $s(\mathcal{X})$ is finite.

Proof of statement (c) of Theorem 1. By the Poincaré-Hopf Theorem a vector field on $\mathbb{S}^{2}$ with a finite number of singular points, satisfies that the sum of the indices of its singular points is +2 , see for instante [4]. 
Under the assumptions all the indices are 1 or -1 . Suppose that $\mathcal{X}$ has $s$ singular points with the index +1 , then the number of the singular points with index -1 is $s-2$. This yields that the vector field $\mathcal{X}$ on $\mathbb{S}^{2}$ has at most $2 s-2$ isolated singular points. Now using statement (b) of Theorem 1, it follows statement (c) of the same theorem.

Proof of statement $(d)$ of Theorem 1. Without loss of generality we shall work with the great circle $\{z=0\} \cap \mathbb{S}^{2}$. Under the stereographic projection at the point $(0,0,1)$, the phase portrait of the vector field $\mathcal{X}$ on $\mathbb{S}^{2} \backslash(0,0,1)$ is topologically equivalent to the one of system (10) with (11) in $\mathbb{R}^{2}$, and the great circle goes over the unite circle $\mathbb{S}^{1}: u^{2}+v^{2}=1$ of $\mathbb{R}^{2}$.

Suppose that the great circle $\mathbb{S}^{1}$ contacts the orbits of the polynomial differential system $(10)$ at $(u, v)$. Then it follows from (14) that

$$
u \dot{u}+v \dot{v}=u \bar{P}(u, v)+v \bar{Q}(u, v)+\left(u^{2}+v^{2}\right) \bar{R}(u, v)=\frac{1}{2}\left(u^{2}+v^{2}+1\right) \bar{R}(u, v) .
$$

So every contact point to $\mathbb{S}^{1}$ satisfies

$$
\bar{R}(u, v)=0, \quad u^{2}+v^{2}=1 .
$$

Since

$$
\left.\bar{R}(u, v)\right|_{(u, v) \in \mathbb{S}^{1}}=\sum_{i+j=0}^{n} 2^{n} r_{i j 0} u^{i} v^{j}
$$

is a polynomial of degree $n$, statement (d) of Theorem 1 follows using the Bezout Theorem.

\section{Proof of Theorem 2}

In this section we study quadratic polynomial vector fields on $\mathbb{S}^{2}$. The next result will be very useful.

Lemma 4. Let $\mathcal{X}$ be a quadratic polynomial vector field in $\mathbb{R}^{3}$ given by (1). Then $\mathcal{X}$ is a quadratic polynomial vector field on $\mathbb{S}^{2}$ if and only if the differential system associated to $\mathcal{X}$ can be written as

$$
\begin{aligned}
\dot{x}= & P(x, y, z)=a_{0}+a_{1} y+a_{2} z-a_{0} x^{2}+a_{3} y^{2}+a_{4} z^{2}+a_{5} x y \\
& +a_{6} x z+a_{7} y z \\
\dot{y}= & Q(x, y, z)=b_{0}-a_{1} x+b_{1} z-\left(a_{5}+b_{0}\right) x^{2}-b_{0} y^{2}+b_{2} z^{2} \\
& -\left(a_{0}+a_{3}\right) x y+b_{3} x z+b_{4} y z \\
\dot{z}= & R(x, y, z)=c_{0}-a_{2} x-b_{1} y-\left(a_{6}+c_{0}\right) x^{2}-\left(b_{4}+c_{0}\right) y^{2}-c_{0} z^{2} \\
& -\left(a_{7}+b_{3}\right) x y-\left(a_{0}+a_{4}\right) x z-\left(b_{0}+b_{2}\right) y z .
\end{aligned}
$$

Proof. Let

$$
x P(x, y, z)+y Q(x, y, z)+z R(x, y, z)-\left(x^{2}+y^{2}+z^{2}-1\right) K(x, y, z) \equiv 0,
$$

where $P, Q, R$ are defined as (9) with $n=2$ and $K(x, y)=\sum_{i+j=0}^{1} k_{i j k} x^{i} y^{j} z^{k}$. Solving this equation and renaming the coefficients of (1), the lemma follows. 
For system (19), we have

$$
\begin{aligned}
\bar{P}(u, v)= & a_{0}-a_{2}+a_{4}-2 a_{6} u+2\left(a_{1}-a_{7}\right) v-2\left(a_{0}+a_{4}\right) u^{2}+4 a_{5} u v \\
& +2\left(a_{0}+2 a_{3}-a_{4}\right) v^{2}+2 a_{6} u^{3}+2\left(a_{1}+a_{7}\right) u^{2} v+2 a_{6} u v^{2} \\
& +2\left(a_{1}+a_{7}\right) v^{3}+\left(a_{0}+a_{2}+a_{4}\right)\left(u^{2}+v^{2}\right)^{2}, \\
\bar{Q}(u, v)= & b_{0}-b_{1}+b_{2}-2\left(a_{1}+b_{3}\right) u-2 b_{4} v-2\left(2 a_{5}+b_{0}+b_{2}\right) u^{2} \\
& -4\left(a_{0}+a_{3}\right) u v-2\left(b_{0}+b_{2}\right) v^{2}-2\left(a_{1}-b_{3}\right) u^{3}+2 b_{4} u^{2} v \\
& -2\left(a_{1}-b_{3}\right) u v^{2}+2 b_{4} v^{3}+\left(b_{0}+b_{1}+b_{2}\right)\left(u^{2}+v^{2}\right)^{2}, \\
\bar{R}(u, v)= & 2\left(a_{0}-a_{2}+a_{4}\right) u+2\left(b_{0}-b_{1}+b_{2}\right) v-4 a_{6} u^{2}-4\left(a_{7}+b_{3}\right) u v \\
& -4 b_{4} v^{2}-2\left(a_{0}+a_{2}+a_{4}\right) u^{3}-2\left(b_{0}+b_{1}+b_{2}\right) u^{2} v \\
& -2\left(a_{0}+a_{2}+a_{4}\right) u v^{2}-2\left(b_{0}+b_{1}+b_{2}\right) v^{3} .
\end{aligned}
$$

We shall give the proof of statement (a) of Theorem 2 at the end of this section.

Proof of statement (b) of Theorem 2. By assumptions $\mathbb{S}^{1}=\{z=0\} \cap \mathbb{S}^{2}$ is an invariant great circle of system $\mathcal{X}$. By Lemma 4 the vector field $\mathcal{X}$ can be written as (19). Under the stereographic projection at the point $(0,0,1)$ the vector field $\mathcal{X}$ becomes system (10) and the great circle $\{z=0\} \cap \mathbb{S}^{2}$ is reduced to the unit circle $\mathbb{S}^{1}: u^{2}+v^{2}=1$ of $\mathbb{R}^{2}$. Note that we denote by $\mathbb{S}^{1}$ the great circle $\{z=0\} \cap \mathbb{S}^{2}$ and the unit circle $u^{2}+v^{2}=1$, of course they are different objects, but they are diffeomorphic through the stereographic projection at the point $(0,0,1)$.

It follows from (17) and (20) that $\mathbb{S}^{1}$ is an invariant circle of (10) if and only if $\bar{R}(u, v)$ is divisible by $u^{2}+v^{2}-1$, i.e. if and only if

$$
a_{2}=a_{6}=b_{1}=b_{4}=0, b_{3}=-a_{7},
$$

holds. Putting conditions (21) into (19) we obtain statement (b.1) of Theorem 2.

The invariant great circle $\mathbb{S}^{1}$ is a periodic orbit of $\mathcal{X}$ if and only if there is no singular point on it. It follows from (4), (10) and $(20)$ that if $(\cos \theta, \sin \theta) \in \mathbb{S}^{1}$, then

$$
\begin{aligned}
& \mathcal{P}(\cos \theta, \sin \theta)=4 \sin \theta\left(a_{1}+a_{5} \cos \theta+\left(a_{0}+a_{3}\right) \sin \theta\right), \\
& \mathcal{Q}(\cos \theta, \sin \theta)=-4 \cos \theta\left(a_{1}+a_{5} \cos \theta+\left(a_{0}+a_{3}\right) \sin \theta\right) .
\end{aligned}
$$

Therefore $\mathbb{S}^{1}$ is a periodic orbit if and only if the following three systems

$$
\begin{array}{ll}
v=0, & a_{1}+a_{5} u+\left(a_{0}+a_{3}\right) v=0, u^{2}+v^{2}=1, \\
u=0, & a_{1}+a_{5} u+\left(a_{0}+a_{3}\right) v=0, u^{2}+v^{2}=1, \\
& a_{1}+a_{5} u+\left(a_{0}+a_{3}\right) v=0, u^{2}+v^{2}=1,
\end{array}
$$

have no solution. This is equivalent to (5). The statement (b.2) follows.

It is well know that a periodic orbit $(u(t), v(t))$ of period $T$ is a hyperbolic limit cycle if and only if

$$
I=\int_{0}^{T}\left(\frac{\partial \mathcal{P}}{\partial u}+\frac{\partial \mathcal{Q}}{\partial v}\right)(u(t), v(t)) d t \neq 0
$$

Let $(u, v)=(\cos \theta, \sin \theta)$. Since $d t=d u / \mathcal{P}(u, v)$, we have

$$
\begin{aligned}
\left.I\right|_{\mathbb{S}^{1}} & =\oint_{\mathbb{S}^{1}} \frac{\mathcal{P}_{u}+\mathcal{Q}_{v}}{\mathcal{P}(u, v)} d u=-\int_{0}^{2 \pi} \frac{\sin \theta\left(\mathcal{P}_{u}(\cos \theta, \sin \theta)+\mathcal{Q}_{v}(\cos \theta, \sin \theta)\right)}{\mathcal{P}(\cos \theta, \sin \theta)} d \theta \\
& =\int_{0}^{2 \pi} \frac{\left(2 a_{0}+a_{3}+a_{4}\right) \cos \theta+\left(b_{0}+b_{2}-a_{5}\right) \sin \theta}{a_{1}+a_{5} \cos \theta+\left(a_{0}+a_{3}\right) \sin \theta} d \theta \\
& =\frac{-2 \pi\left(a_{5}\left(a_{0}+a_{4}\right)+\left(a_{0}+a_{3}\right)\left(b_{0}+b_{2}\right)\right)}{\sqrt{a_{1}^{2}-a_{5}^{2}-\left(a_{0}+a_{3}\right)^{2}}\left(\left|a_{1}\right|+\sqrt{a_{1}^{2}-a_{5}^{2}-\left(a_{0}+a_{3}\right)^{2}}\right)},
\end{aligned}
$$


where we use the fact that $\left|a_{1}\right|>\left|a_{5}\right|$ (see (5)). Hence $\mathbb{S}^{1}$ is a hyperbolic limit cycle of system (10) if and only if $\left.I\right|_{\mathbb{S}^{1}} \neq 0$ and (5) holds. So statement (b.3) is proved.

Finally we are going to prove (b.4). We note that $\left.I\right|_{\mathbb{S}^{1}}=0$ if and only if $a_{5}\left(a_{0}+\right.$ $\left.a_{4}\right)+\left(a_{0}+a_{3}\right)\left(b_{0}+b_{2}\right)=0$. Suppose that $(5)$ holds and $\left.I\right|_{\mathbb{S}^{1}}=0$. Then by direct computation, we have

(26) $u \mathcal{P}(u, v)+v \mathcal{Q}(u, v)=-\left(\left(a_{0}+a_{4}\right) u+\left(b_{0}+b_{2}\right) v\right)\left(u^{2}+v^{2}+1\right)\left(u^{2}+v^{2}-1\right)$,

which implies that the coordinates of the singular points of system (10) under the assumptions satisfy $\left(a_{0}+a_{4}\right) u+\left(b_{0}+b_{2}\right) v=0$. If $a_{0}+a_{4} \neq 0$, then

$$
\left.\left(\frac{\partial \mathcal{P}}{\partial u}+\frac{\partial \mathcal{Q}}{\partial v}\right)\right|_{a_{5}=-\frac{\left(a_{0}+a_{3}\right)\left(b_{0}+b_{2}\right)}{a_{0}+a_{4}}, u=-\frac{b_{0}+b_{2}}{a_{0}+a_{4}} v}=0 .
$$

If $a_{0}+a_{4}=0, b_{0}+b_{2} \neq 0$, then $a_{5}\left(a_{0}+a_{4}\right)+\left(a_{0}+a_{3}\right)\left(b_{0}+b_{2}\right)=0$ implies $a_{0}+a_{3}=0$. We have

$$
\left.\left(\frac{\partial \mathcal{P}}{\partial u}+\frac{\partial \mathcal{Q}}{\partial v}\right)\right|_{v=0, a_{3}=-a_{0}, a_{4}=-a_{0}}=0 .
$$

Since the coordinates of the singular points of system (10) under these assumptions satisfy $\left(a_{0}+a_{4}\right) u+\left(b_{0}+b_{2}\right) v=0$, the above equation is also equal to zero if $\left(u^{*}, v^{*}\right)$ is a singular point of system (10). This proves (b.4) except perhaps if $(0,0,1)$ is a singular point of $\mathcal{X}$, but this is not the case because the origin of system (12) is a singular point if and only if $a_{0}+a_{4}=b_{0}+b_{2}=0$, which by assumption it is not the case.

Under the assumptions of statement (b.4) of Theorem 2, if a singular point of $\mathcal{X}$ is focus, then it is non-hyperbolic.

Proof of statement (c) of Theorem 2. Suppose that a homoclinic loop of $\mathcal{X}$ on $\mathbb{S}^{2}$ contains an arc of a great circle. Without loss of generality we assume that this great circle is $\{z=0\} \cap \mathbb{S}^{2}$. It follows from statement (b) of Theorem 2 that if $\{z=0\} \cap \mathbb{S}^{2}$ is an invariant great circle of $\mathcal{X}$, then $\mathcal{X}$ can be written as (4). Since the coordinates of the singular points on $\{z=0\} \cap \mathbb{S}^{2}$ are determined by equations (22), (23) and (24), the number of the singular points on the great circle is an even number, which implies that $\{z=0\} \cap \mathbb{S}^{2}$ is not a homoclinic loop of $\mathcal{X}$.

Statement $(c)$ of Theorem 2 also holds for the vector fields on $\mathbb{S}^{2}$ induced by homogeneous vector fields of degree two in $\mathbb{R}^{3}$, see $[8,14]$.

The statements (d) and (e) of Theorem 2 show some of the differences between homogeneous and non-homogeneous quadratic polynomial vector fields on $\mathbb{S}^{2}$.

Proof of statement (d) of Theorem 2. Consider the vector field (4). If $a_{0}=a_{3}=0$, $a_{1}=2$ and $a_{4}=a_{5}=1$, then (6) becomes $2 / \sqrt{3}-1$, and (5) holds. So statement (d) of Theorem 2 follows from statement (b) of the same theorem.

Proof of statement (e) of Theorem 2. Suppose that (4) is a quadratic homogeneous polynomial vector field (i.e. $a_{0}=a_{1}=b_{0}=c_{0}=0$ ) having $\mathbb{S}^{1}$ is an invariant great circle. Since $\left(a_{0}+a_{3}\right)^{2}-a_{1}^{2}+a_{5}^{2}=a_{3}^{2}+a_{5}^{2} \geq 0$, (5) does not holds. Therefore from statement (b.2) of Theorem 2 it follows statement (e) of the same theorem. 
Statement (e) of Theorem 2 also holds for the vector fields on $\mathbb{S}^{2}$ induced by homogeneous vector field of degree two in $\mathbb{R}^{3}$, see [8]. We also note that statement (e) of Theorem 2 was proved in [10] using different arguments.

Proof of statement $(f)$ of Theorem 2. Consider system (19) with $a_{0}=a_{2}=a_{3}=$ $a_{6}=0, a_{1}=2, a_{4}=a_{5}=1, a_{7}=10, b_{0}=b_{1}=b_{2}=b_{4}=0$ and $b_{3}=-10$. It follows from system (10) that the stereographic projection of system (19) is given by

$$
\begin{aligned}
& \dot{u}=1-16 v+4 u v-2 v^{2}+24 u^{2} v+24 v^{3}-u^{4}+v^{4}=\mathcal{P}(u, v) \\
& \dot{v}=-2 u\left(-8+2 u-v+12 u^{2}+12 v^{2}+u^{2} v+v^{3}\right)=\mathcal{Q}(u, v) .
\end{aligned}
$$

By direct computation $\mathcal{P}(0, v)=1-16 v-2 v^{2}+24 v^{3}+v^{4}$ and $\mathcal{P}(0, \pm \infty)=+\infty$, $\mathcal{P}(0,-1)=-8, \mathcal{P}(0,0)=1, \mathcal{P}(0,1 / 2)=-71 / 16$ and $\mathcal{P}(0,1)=8$. This implies that system $(27)$ has at least four singular points on the line $u=0$ and three of them are inside the circle $u^{2}+v^{2}=1$.

The system we consider has the form as system (4). Since the given coefficients of system (19) satisfy (5) and (6), it follows from statement (b) of Theorem 2 that the great circle $\{z=0\} \cap \mathbb{S}^{2}$ is a limit cycle of system (19) with the given coefficients. On the other hand, the equality $u \mathcal{P}(u, v)+v \mathcal{Q}(u, v)=-u\left(u^{2}+v^{2}-1\right)\left(u^{2}+v^{2}+1\right)$ shows that the coordinates of singular points of system (27) satisfy either $u=0$, or $u^{2}+v^{2}=1$. Since $u^{2}+v^{2}=1$ is the stereographic projection of the great circle $\{z=0\} \cap \mathbb{S}^{2}$ which is a limit cycle, there is no singular point on $u^{2}+v^{2}=1$. Therefore system (19) with the given coefficients has only four singular points on $\mathbb{S}^{2}$. One is the singular point $(0,0,1)$ of the northern hemisphere and the other three are in the southern hemisphere.

Proof of statement $(g)$ of Theorem 2. Here we use similar arguments to those of [8]. Without loss of generality we suppose that $p=(0,0,-1)$ is a singular point of $\mathcal{X}$. Then $\mathcal{X}$ becomes system (10) under the stereographic projection $\pi^{-1}$ at the point $p$. Since $\mathcal{X}$ is a quadratic homogeneous polynomial vector field, it follows from Lemma 4 that $\mathcal{X}$ can be written as (19) with $a_{0}=a_{1}=a_{2}=a_{4}=b_{0}=b_{1}=b_{2}=c_{0}=0$.

A direct computation shows that $(0,0,1)$ is also a singular point of $\mathcal{X}$.

The origin $q=(0,0)$ is a singular point of the stereographic projection given by system (10). Assume that $q$ is a hyperbolic saddle or a hyperbolic proper node of (10). Then the linear part of system (10) at $q$

$$
\left(\begin{array}{ll}
-2 a_{6} & -2 a_{7} \\
-2 b_{3} & -2 b_{4}
\end{array}\right)
$$

has two different real eigenvalues. This implies

$$
\left(a_{6}+b_{4}\right)^{2}-4\left(a_{6} b_{4}-a_{7} b_{3}\right)=\left(b_{4}-a_{6}\right)^{2}+4 a_{7} b_{3}>0 .
$$

Consider the straight line $L=v-k u=0$. We have

$$
\left.\frac{d L}{d t}\right|_{L=0}=2\left(b_{3}+\left(b_{4}-a_{6}\right) k-a_{7} k^{2}\right) u\left(\left(1+k^{2}\right) u^{2}-1\right)-4\left(a_{5}+a_{3} k\right)\left(1+k^{2}\right) u^{2} .
$$

Since (28) holds, the equation $b_{3}+\left(b_{4}-a_{6}\right) k-a_{7} k^{2}=0$ has two real zeros for $k$ if $a_{7} \neq 0$, and one real zero if $a_{7}=0$. Let $k$ be one zero of the above equation. Then we have

$$
\left.\frac{d L}{d t}\right|_{L=0}=-4\left(a_{5}+a_{3} k\right)\left(1+k^{2}\right) u^{2} .
$$


If $d L /\left.d t\right|_{L=0}$ does not change sign and $d L /\left.d t\right|_{L=0} \not \equiv 0$, then all orbits pass through $L$ in the same direction. If $d L /\left.d t\right|_{L=0} \equiv 0$, then $L$ is an invariant straight line of system $(10)$. Since $\pi(L)$ is a great circle on $\mathbb{S}^{2}$, statement (f) of Theorem 2 follows.

Statement $(\mathrm{g})$ of Theorem 2 shows that if a quadratic homogeneous polynomial vector field $\mathcal{X}$ on $\mathbb{S}^{2}$ has either a hyperbolic saddle or a hyperbolic proper node, then a periodic orbit is entirely contained in a hemisphere. This assertion also holds for the vector fields on $\mathbb{S}^{2}$ induced by homogeneous quadratic polynomial vector fields of in $\mathbb{R}^{3}$, see $[2]$.

Proof of statement $(h)$ of Theorem 2. There exists polynomial vector fields on $\mathbb{S}^{2}$ such that $\mathbb{S}^{2}$ is completely filled by centers and their families of period annulus. For example the linear differential system of the form

$$
\dot{x}=-y, \quad \dot{y}=x, \quad \dot{z}=0,
$$

has the two poles of the sphere $\mathbb{S}^{2}$ as singular points and all the parallels as periodic orbits. The same occurs for the quadratic polynomial vector field

$$
\dot{x}=-y(z-2), \quad \dot{y}=x(z-2), \quad \dot{z}=0 .
$$

So statement $(g)$ of Theorem 2 is proved. But we provide a big family of quadratic polynomial vector fields on $\mathbb{S}^{2}$ with the same topological equivalent phase portrait.

In statement (b.4) of Theorem 2 we suppose that $\left(a_{0}+a_{4}\right)^{2}+\left(b_{0}+b_{2}\right)^{2} \neq 0$. What happen if $\left(a_{0}+a_{4}\right)^{2}+\left(b_{0}+b_{2}\right)^{2}=0$ ? We will show that all these quadratic polynomial vector fields on $\mathbb{S}^{2}$ have the phase portrait described in statement $(\mathrm{g})$ of Theorem 2.

Suppose that the vector field (4) satisfies (5), and that additionally $a_{4}=-a_{0}$ and $b_{2}=-b_{0}$, then (4) becomes

$$
\begin{aligned}
& \dot{x}=P(x, y, z)=a_{0}+a_{1} y-a_{0} x^{2}+a_{3} y^{2}-a_{0} z^{2}+a_{5} x y+a_{7} y z \\
& \dot{y}=Q(x, y, z)=b_{0}-a_{1} x-\left(a_{5}+b_{0}\right) x^{2}-b_{0} y^{2}-b_{0} z^{2}-\left(a_{0}+a_{3}\right) x y-a_{7} x z \\
& \dot{z}=R(x, y, z)=-c_{0}\left(x^{2}+y^{2}+z^{2}-1\right) .
\end{aligned}
$$

The stereographic projection of $(29)$ at the point $(0,0,1)$ provides the differential system

$$
\begin{aligned}
& \dot{u}=2 v\left(a_{1}-a_{7}+2 a_{5} u+2\left(a_{0}+a_{3}\right) v+\left(a_{1}+a_{7}\right)\left(u^{2}+v^{2}\right)\right), \\
& \dot{v}=-2 u\left(a_{1}-a_{7}+2 a_{5} u+2\left(a_{0}+a_{3}\right) v+\left(a_{1}+a_{7}\right)\left(u^{2}+v^{2}\right)\right) .
\end{aligned}
$$

If $a_{1}+a_{7} \neq 0$ and $a_{1}^{2}-a_{7}^{2}-a_{5}^{2}-\left(a_{0}+a_{3}\right)^{2}>0$, we have

$$
\begin{aligned}
& a_{1}-a_{7}+2 a_{5} u+2\left(a_{0}+a_{3}\right) v+\left(a_{1}+a_{7}\right)\left(u^{2}+v^{2}\right) \\
= & \left(a_{1}+a_{7}\right)\left(\left(u+\frac{a_{5}}{a_{1}+a_{7}}\right)^{2}+\left(v+\frac{a_{0}+a_{3}}{a_{1}+a_{7}}\right)^{2}+\frac{a_{1}^{2}-a_{7}^{2}-a_{5}^{2}-\left(a_{0}+a_{3}\right)^{2}}{\left(a_{1}+a_{7}\right)^{2}}\right) \\
\neq & 0,
\end{aligned}
$$

which implies that system (30) has the unique singular point at the origin which is a center. Therefore $(0,0,-1)$ is a center of system $(29)$.

By the same arguments we conclude that the $(0,0,1)$ is also a center of system (29). Hence $\mathbb{S}^{2}$ is completely filled by two centers at $(0,0, \pm 1)$ and their periodic orbits. 
Proof of statement $(i)$ of Theorem 2. Suppose that a quadratic vector field $\mathcal{X}$ on $\mathbb{S}^{2}$ has 6 isolated singular points at $( \pm 1,0,0),(0, \pm 1,0)$ and $(0,0, \pm 1)$, respectively. It follows from Lemma 4 that $\mathcal{X}$ can be written as

$$
\begin{aligned}
& \dot{x}=a_{0}-a_{0} x^{2}-a_{0} y^{2}-a_{0} z^{2}+a_{7} y z, \\
& \dot{y}=b_{0}-b_{0} x^{2}-b_{0} y^{2}-b_{0} z^{2}+b_{3} x z, \\
& \dot{z}=c_{0}-c_{0} x^{2}-c_{0} y^{2}-c_{0} z^{2}-\left(a_{7}+b_{3}\right) x y .
\end{aligned}
$$

Doing the change $2 t=\tau$, the stereographic projections of system (31) at the points $(0,0,1)$ and $(0,0,-1)$ are given by

$$
\begin{aligned}
& \dot{u}=v\left(-a_{7}-\left(a_{7}+2 b_{3}\right) u^{2}+a_{7} v^{2}\right) \\
& \dot{v}=u\left(-b_{3}+b_{3} u^{2}-\left(2 a_{7}+b_{3}\right) v^{2}\right)
\end{aligned}
$$

and

$$
\begin{aligned}
& \dot{u}=v\left(a_{7}+\left(a_{7}+2 b_{3}\right) u^{2}-a_{7} v^{2}\right), \\
& \dot{v}=u\left(b_{3}-b_{3} u^{2}+\left(2 a_{7}+b_{3}\right) v^{2}\right),
\end{aligned}
$$

respectively, where the dot now denotes derivative with respect to $\tau$. If $a_{7} b_{3}=0$, then system (32) and (33) has non-isolated singular points. Therefore we suppose $a_{7} b_{3} \neq 0$.

By direct computation we know that the linearized system of (32) and (33) at each singular point has two non-zero eigenvalues. So the topological index at every one of its singular points is \pm 1 (for more details see [4]). Since a vector field on $\mathbb{S}^{2}$ with a finite number of singular points satisfies that the sum of their indices is 2 (see the Poincaré-Hopf Theorem for instance in [4]), system (31) on $\mathbb{S}^{2}$ has two saddles whose indices are -1 and four antisaddles with indices 1 . Without loss of generality we assume that $(0,0,-1)$ is a saddle. This implies $a_{7} b_{3}>0$. Moreover we take $a_{7}=1$ and $b_{3}>0$. By direct computations we check that system (33) has a saddle at $(0,0)$, and that system $(32)$ has the first integral

$$
H(u, v)=\frac{-b_{3} u^{2}+v^{2}}{\left(1+u^{2}+v^{2}\right)^{2}},
$$

the four centers $(0, \pm 1)$ and $( \pm 1,0)$, and the saddle $(0,0)$.

It follows from (34) that system (32) has two invariant straight lines $v= \pm \sqrt{b_{3}} u$, which correspond to two invariant great circles $\left\{(x, y) \mid y= \pm \sqrt{b_{3}} x\right\} \cap \mathbb{S}^{2}$ of system (31). So the proof of statement (i) of Theorem 2 is completed.

Proof of statement (a) of Theorem 2. From statement (c) of Theorem 1 we know that $s(\mathcal{X}) \leq 6$. Since the quadratic polynomial vector field $\mathcal{X}$ on $\mathbb{S}^{2}$ of statement (i) of Theorem 2 has exactly 6 singular points, statement (a.1) of Theorem 2 is proved.

By statement (a.1) of Theorem 2 a quadratic polynomial vector field $\mathcal{X}$ such that all the indices of its singular points are \pm 1 has at most 6 singular points. Since centers have index 1 and the sum of all the indices is always 2 (by the PoincaréHopf Theorem), the first part of statement (a.2) of Theorem 2 follows. The bound of 4 centers is reached by statement (i) of Theorem 2. Hence statement (a.2) of Theorem 2 is completely proved.

Of course the proof of the first part of statement (a.3) of Theorem 2 is the same that the proof of the first part of statement (a.2). Now we shall provide a quadratic polynomial vector field on $\mathbb{S}^{2}$ with 4 foci. 
Consider the quadratic polynomial vector field on $\mathbb{S}^{2}$ given by

$$
\begin{aligned}
& P(x, y, z)=a_{0}-a_{0} x^{2}+a_{3} y^{2}-a_{0} z^{2}+a_{7} y z, \\
& Q(x, y, z)=b_{0}-b_{0} x^{2}-b_{0} y^{2}-b_{0} z^{2}-\left(a_{0}+a_{3}\right) x y+b_{3} x z+b_{4} y z, \\
& R(x, y, z)=c_{0}-c_{0} x^{2}-\left(b_{4}+c_{0}\right) y^{2}-c_{0} z^{2}-\left(a_{7}+b_{3}\right) x y .
\end{aligned}
$$

Doing the change $2 t=\tau$ the stereographic projections of system (35) at the points $(0,0,1)$ and $(0,0,-1)$ are given by

$$
\begin{aligned}
& \dot{u}=v\left(-a_{7}+2\left(a_{0}+a_{3}\right) v-\left(a_{7}+2 b_{3}\right) u^{2}-2 b_{4} u v+a_{7} v^{2}\right), \\
& \dot{v}=-b_{3} u-b_{4} v-2\left(a_{0}+a_{3}\right) u v+b_{3} u^{3}+b_{4} u^{2} v-\left(2 a_{7}+b_{3}\right) u v^{2}-b_{4} v^{3},
\end{aligned}
$$

and

$$
\begin{aligned}
& \dot{u}=v\left(a_{7}+2\left(a_{0}+a_{3}\right) v+\left(a_{7}+2 b_{3}\right) u^{2}+2 b_{4} u v-a_{7} v^{2}\right), \\
& \dot{v}=b_{3} u+b_{4} v-2\left(a_{0}+a_{3}\right) u v-b_{3} u^{3}-b_{4} u^{2} v+\left(2 a_{7}+b_{3}\right) u v^{2}+b_{4} v^{3},
\end{aligned}
$$

respectively. It follows from (36) and (37) that, if $b_{4}^{2}+4 a_{7} b_{3}<0,\left(a_{0}+a_{3}\right)^{2}-$ $4\left(a_{7}+b_{3}\right) b_{3}<0, b_{4} \neq 0$ and $a_{0}+a_{3} \neq 0$, then $( \pm 1,0,0)$ and $(0,0, \pm 1)$ are foci of system (35). Hence statement (a.3) of Theorem 2 is proved.

Since $s(\mathcal{X}) \leq 6$, saddles have index -1 and the sum of all the indices is always 2 , the first part of statement (a.4) of Theorem 2 follows. The bound of 2 saddles is reached by statement (i) of Theorem 2. Hence statement (a.4) of Theorem 2 is proved.

\section{ACKNOWLEDGEMENTS}

We thank to the referee some comments which help us to improve the presentation of this paper.

The second author wants to express his thanks to the Departament de Matemàtiques of the Universitat Autònoma de Barcelona for the hospitality and support during the period in which this paper was started.

The first author is partially supported by a DGICYT/FEDER grant number MTM2008-03437 and by a CICYT grant number 2009SGR 410. The second author is partially supported by the Spanish grant SAB2005-0029, NSF of China (No. 10871214) and Program for New Century Excellent Talents in University.

\section{REFERENCES}

11] C. Coleman, A certain class of integral curves in 3-space, Ann. Math. 69 (1959), 678-685.

[2] M.I.T. CAmacho, Geometric properties of homogeneous vector fields of degree two in $R^{3}$, Trans. Amer. Math. Soc 268 (1981), 79-101.

[3] D. Cox, J. Little And D. O'Shea, Using Algebraic Geometry, Springer-Verlag, 1998.

[4] F. Dumortier, J. Llibre, J. C. Artés, Qualititive theory of planar differential systems, Springer, 2006.

[5] J. ÉCALLE, Introduction aux fonctions analysables et preuve constructive de la conjecture de Dulac (French), Hermann, Paris, 1992.

[6] W. Fulton, Algebraic Curves. An Introduction to Algebraic Geometry, Benjamin, New York, 1969.

[7] Yu. Ilyashenko, Finiteness theorems for limit cycles, Amer. Math. Soc., Providence, RI, 1991.

[8] Zhaojun Liang, Periodic orbits of homogeneous vector fields of degree two in $\mathbb{R}^{3}$, Dynamical systems (Tianjin, 1990/1991), Nankai Ser. Pure Appl. Math. Theoret. Phys. 4, World Sci. Publ. Edge. 1993, pp. 111-125.

[9] J. Llibre AND C. PEssoA, Invariant circles for homogeneous polynomial vector fields on the 2-dimentional sphere, Rend. Circ. Mat. Palermo 55 (2006), 63-81. 
[10] J. Llibre and C. Pessoa, Homgeneous polynomial vector fields of degree 2 on the 2dimensional sphere, Extracta Math. 21 (2006), 167-190.

[11] J. Llibre And C. PessoA, Phase portraits for quadratic homogeneous polynomial vector fields on $\mathbb{S}^{2}$, preprint.

[12] I.R. Shafaravich, Basic Algebraic Geometry, Springer, 1974.

[13] Sh.R. Sharipov, Classification of integral manifolds of a homogeneous three-dimensional system according to the structure of limit sets, Diff. Uravn. 7 (1971), 355-363.

[14] YANQIAN Ye, Qualitative theory of polynomial differential systems (in Chinese), Modern Mathematics Series, Shanghai Scientific \& Technical Publishers, 1995.

[15] Xinan Zhang, Lansun Chen and Zhaojun Liang, The gloabal topological properties of homogeneous vector fields in $\mathbb{R}^{n}$, Chin. Ann. Math. 20B (1999), 185-194.

1 Departament de Matemàtiques, Universitat Autònoma de Barcelona, 08193 Bellaterra, Barcelona, Catalonia, Spain

E-mail address: jllibre@mat.uab.cat

2 Department of Mathematics, Sun Yat-Sen University, Guangzhou, 510275, People's Republic of China.

E-mail address: mcszyl@mail.sysu.edu.cn 